\title{
Perforación diverticular sigmoidea secundaria a la migración de endoprótesis biliar plástica*
}

\author{
Drs. BEATRIZ DE ANDRÉS A. ${ }^{1}$, FRANCISCA MORENO R. ${ }^{1}$, PATRICIA LEGIDO M. ${ }^{1}$, \\ JOSÉ RABADÁN J. ${ }^{1}$, JUAN BELTRÁN DE HEREDIA R. ${ }^{1}$ \\ Servicio de Cirugía General y del Aparato Digestivo. Hospital Clínico Universitario. \\ Valladolid, España.
}

\begin{abstract}
\section{Sigmoid diverticulum perforation due to plastic biliary stent migration}

Background: Biliary stents are devices that are used to widen narrowed bile ducts, especially in malignant obstructions, although at present its use in benign biliopancreatic diseases is progressively increasing. Endoscopic placement of biliary stents is a well established procedure that is performed daily. Despite its frequency, has a complication rate of $5-10 \%$, the most common are cholangitis and stent obstruction and there are others more rare and serious like pancreatitis, gastrointestinal bleeding, intestinal perforation and biliary stent migration. Case report: We report a 70 -years-old male with a history of plastic biliary stent placement after open cholecystectomy because of choledocholithiasis, who arrived at the Emergency Department with symptoms of intestinal obstruction. After performing imaging studies, there was significant intestinal dilation and a biliary stent in the sigmoid colon. He underwent urgent surgical intervention, having the plastic biliary stent located in a sigmoid diverticulum. We performed removal of the stent and simple suture of the sigmoid diverticular perforation. Conclusion: Sigmoid diverticular perforation secondary to migration of a plastic biliary stent is a rare complication that can occur after placement of a stent in the bile duct. It must be suspected in all acute abdomens accompanied of radiological images demonstrating biliary stent migration because these patients often show atypical symptoms.
\end{abstract}

Key words: Biliary stent, migration, intestinal perforation.

\section{Resumen}

Introducción: Las endoprótesis biliares son dispositivos que se emplean para ampliar las vías biliares estenosadas, especialmente en las obstrucciones malignas, aunque actualmente su uso está aumentando progresivamente en las enfermedades benignas biliopancreáticas. La colocación endoscópica de las endoprótesis biliares es un procedimiento bien establecido que se realiza diariamente, aunque presenta una tasa de complicaciones de 5-10\%, siendo las más frecuentes la colangitis y la obstrucción de la propia endoprótesis, existiendo otras más raras y graves como la pancreatitis, la hemorragia digestiva, la perforación intestinal y la migración de la endoprótesis biliar. Caso clínico: Varón de 70 años de edad con antecedentes de colocación de

\footnotetext{
*Recibido el 10 de diciembre de 2012 y aceptado para publicación el 23 de enero de 2013.
}

Los autores no refieren conflictos de interés ni apoyo financiero.

Correspondencia:

Dra. Beatriz de Andrés A.

C/ Ribera del Carrión 1, 3 B. 47008 Valladolid, España.

beatrizdeandres007@yahoo.es 
endoprótesis biliar plástica tras presentar coledocolitiasis posterior a colecistectomía, que acudió al Servicio de Urgencias con clínica de obstrucción intestinal. Después de realizar radiología simple y ecografía abdominal, se observó importante dilatación intestinal y una endoprótesis biliar en sigma. Se practicó intervención quirúrgica urgente, hallándose endoprótesis biliar plástica enclavada en un divertículo sigmoideo. Se realizó extracción de la endoprótesis y sutura simple de la perforación diverticular sigmoidea. Conclusión: La perforación diverticular sigmoidea secundaria a la migración de una endoprótesis biliar plástica es una complicación poco frecuente que puede aparecer tras la colocación de una endoprótesis en la vía biliar. Dicha perforación debe sospecharse ante todo cuadro de abdomen agudo que se acompaña de imágenes radiológicas que demuestren la migración de la endoprótesis biliar, ya que estos pacientes no suelen presentar una sintomatología típica.

Palabras clave: Endoprótesis biliar, migración, perforación intestinal.

\section{Introducción}

Las endoprótesis biliares son unos dispositivos que se usan para ampliar las vías biliares estrechas o estenosadas, principalmente en las obstrucciones malignas causadas por cáncer pancreático, colangiocarcinoma, cáncer vesicular y cáncer de la ampolla de Vater; pero también se aplican en patología benigna de la vía biliar, como son las fístulas biliares tras cirugía biliar y la coledocolitiasis tras intento fallido de su extracción por vía endoscópica $^{1}$. Existen diversos tipos de endoprótesis: las plásticas, que pueden tener diferentes formas: rectas, tipo monopig-tail y doble pig-tail, de las cuales actualmente las más empleadas son las rectas; y las endoprótesis metálicas autoexpandibles, que se usan principalmente en las estenosis malignas. La colocación endoscópica de las endoprótesis biliares es un procedimiento bien establecido que se realiza diariamente, sin embargo, no está exento de complicaciones, que ocurren entre el 5-10\% de los casos, siendo las más frecuentes la colangitis y la obstrucción de la propia endoprótesis, existiendo otras más raras y graves como la pancreatitis, la hemorragia digestiva, la perforación intestinal y la migración de la endoprótesis biliar².

Presentamos el caso de un paciente con perforación diverticular sigmoidea secundaria a la migración de una endoprótesis biliar plástica, complicación poco frecuente tras la colocación de una endoprótesis en la vía biliar.

\section{Caso clínico}

Paciente varón de 70 años con antecedentes de coledocolitiasis tras colecistectomía, que precisó 2 meses antes de su ingreso hospitalario, la realización de una colangiopancreatografía retrógrada endoscópica (CPRE) con extracción de cálculos, esfinterotomía y colocación de una endoprótesis biliar plástica de 10 French y $9 \mathrm{~cm}$ de longitud. Acudió al Servicio de Urgencias por presentar dolor abdominal difuso, vómitos biliosos y ausencia de tránsito intestinal de
3 días de evolución. A la exploración física presentaba abdomen muy distendido, timpánico, doloroso de forma difusa, sin signos de irritación peritoneal y ruidos intestinales metálicos. En el análisis de sangre destacaba leucocitosis $14.950 / \mu \mathrm{L} \quad(91,5 \%$ neutrófilos), bilirrubina total $1,54 \mathrm{mg} / \mathrm{dL}$, bilirrubina directa $0,57 \mathrm{mg} / \mathrm{dL}$ y sodio $131 \mathrm{mEq} / \mathrm{L}$. La radiología simple abdominal mostró importante distensión de asas intestinales y endoprótesis biliar en el sigma (Figura 1) y en la de bipedestación, múltiples niveles hidroaéreos. Se realizó ecografía abdominal observándose gran dilatación de asas intestinales y colon, compatible con obstrucción intestinal, sin apreciarse líquido libre abdominal. Ante la presencia de un abdomen agudo y la migración de la endoprótesis biliar, se realizó intervención quirúrgica urgente, hallándose perforación de un divertículo sigmoideo causada por el enclavamiento de la endoprótesis biliar plástica en el divertículo (Figura 2). Se realizó

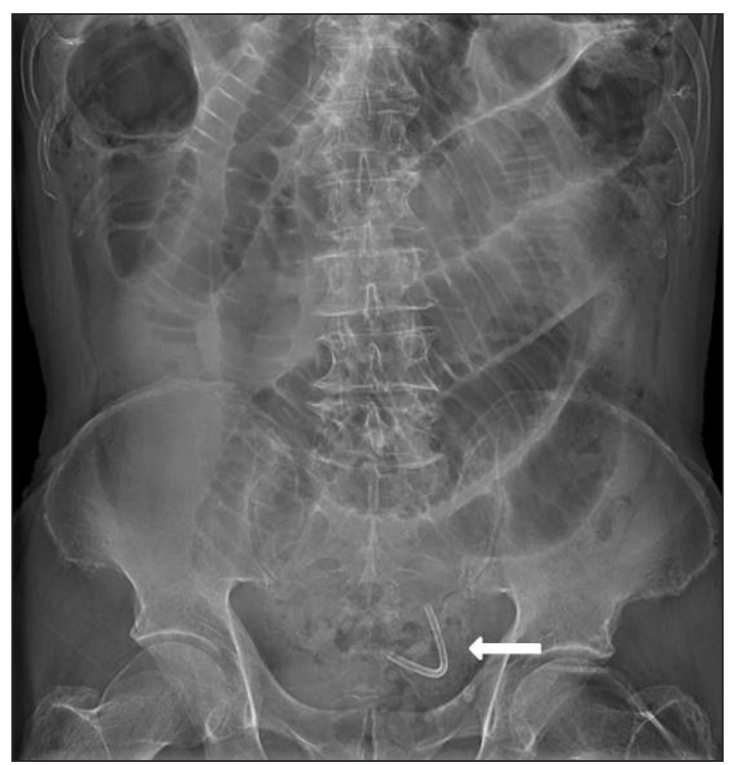

Figura 1. Radiografía simple de abdomen. Se observa dilatación de todo el intestino delgado y la flecha señala la endoprótesis biliar en sigma. 


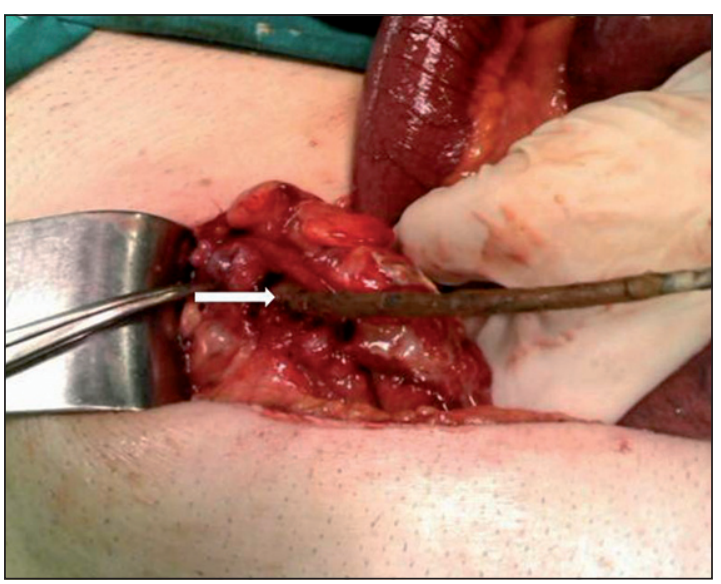

Figura 2. Los hallazgos intraoperatorios muestran la endoprótesis biliar plástica enclavada en un divertículo sigmoideo indicado con la flecha.

extracción de la endoprótesis y sutura simple de la perforación diverticular sigmoidea. El postoperatorio cursó con normalidad.

\section{Discusión}

En la actualidad el empleo de las endoprótesis biliares es muy común y su colocación endoscópica en las vías biliares es un procedimiento bien protocolizado, descrito por primera vez en 1980 por Soehendra y Reynders-Frederix ${ }^{3}$. Las endoprótesis biliares pueden ser de diferentes tipos: plásticas (Teflon, polietileno o poliuretano) y metálicas autoexpandibles. Las endoprótesis plásticas son más baratas y más fáciles de extraer o recambiar, pero tienen más riesgo de obstrucción ${ }^{4}$; su forma es variable, pueden ser rectas, tipo monopig-tail o doble pig-tail, siendo las rectas las más utilizadas, su longitud varía entre 5 y $18 \mathrm{~cm}$ y su diámetro entre 7 y 11,5 French. El objetivo de las endoprótesis biliares es ampliar la luz estenosada o estrecha de las vías biliares manteniendo la permeabilidad de las mismas. Se emplean principalmente en la ictericia obstructiva secundaria a enfermedades malignas como la neoplasia pancreática, el colangiocarcinoma, el cáncer vesicular y el cáncer de la ampolla de Vater; pero también se aplican en patologías benignas como las fístulas biliares tras cirugía biliar y la coledocolitiasis tras intento fallido de la extracción de cálculos en el colédoco ${ }^{1}$. Una vez colocada la endoprótesis biliar debe verificarse su posición correcta por vía endoscópica y fluoroscópica para dar por finalizada la CPRE.

La colocación endoscópica de las endoprótesis biliares presenta una mortalidad inferior al $1 \%$; no está exenta de complicaciones, que ocurren entre el $5-10 \%$ de los casos, siendo las más frecuentes la colangitis y la obstrucción de la propia endoprótesis; otras más raras y graves son la pancreatitis, la hemorragia digestiva, la perforación intestinal y la migración de la endoprótesis biliar ${ }^{2}$. Estas complicaciones suelen producirse inmediatamente $\mathrm{o}$ a corto plazo, siendo las complicaciones a largo plazo las más difíciles de diagnosticar y de tratar, como es el caso de la perforación diverticular de sigma por la migración de la endoprótesis biliar plástica que presentamos; por lo que a todos los pacientes portadores de endoprótesis biliares plásticas debe realizárseles un seguimiento cercano, extrayéndoles o recambiándoles periódicamente la endoprótesis para evitar la rotura, obstrucción, infección o migración de la misma; especialmente en aquellos pacientes que presenten factores de riesgo como hernias abdominales, adherencias intestinales y diverticulosis colónica. Se aconseja realizar el recambio de la endoprótesis biliar a los tres meses ${ }^{1}$, aunque se puede prolongar este período hasta los seis meses ${ }^{5}$; y para evitar su migración se debe colocar la endoprótesis a través del esfínter de Oddi ${ }^{6}$, no debiendo sobrepasar la ampolla más de $1 \mathrm{~cm}^{7}$. En nuestro paciente no se pudo realizar el control rutinario de la endoprótesis ya que se complicó a los dos meses de su colocación, por lo que fue un hallazgo casual observarla en el sigma.

La migración de las endoprótesis biliares ocurre entre el 5-10\% de los pacientes ${ }^{4,8-13}$, aunque existen discrepancias, ya que Belyaev ${ }^{14}$, refiere una frecuencia menor del $5 \%$ y Bagul $^{2}$, mayor del $10 \%$ porque considera que la mayoría de las endoprótesis que migran al intestino, pasan a través de él sin incidencias. Las migraciones pueden ser proximales, desplazándose la endoprótesis en la propia vía biliar o en el conducto pancreático; o distales, alcanzando el intestino delgado y el colon. Según Johanson ${ }^{13}$, existe un elevado riesgo de migración proximal en las vías biliares cuando se asocia a estenosis malignas como el colangiocarcinoma, a las endoprótesis de mayor diámetro y a las endoprótesis de corta longitud; y la migración distal ocurre más frecuentemente en la estenosis papilar, no hallando otros factores de riesgo significativos en la migración distal. Se ha observado que la migración distal es más frecuente en las estenosis benignas que en las malignas, posiblemente debido a que las estenosis benignas son menos estrechas a causa de la desaparición de la inflamación y del edema de la mucosa de las vías biliares con el paso del tiempo, a diferencia de las estenosis malignas donde el tejido tumoral ancla la endoprótesis previniendo su migración ${ }^{2,11,15}$. Arhan ${ }^{11}$, ha observado que la migración protésica es menos frecuente en las estenosis postcolecistectomía que 
en otras estenosis benignas, excepto en la colangitis esclerosante primaria, probablemente secundario a la existencia del tejido fibroso que forma la estenosis. Al igual que Johanson ${ }^{13}$, ha hallado que en las estenosis biliares benignas las endoprótesis cortas tienden a migrar proximalmente, y las largas distalmente; sin encontrar diferencias significativas en relación con el calibre de la endoprótesis y la frecuencia y dirección de la migración.

Existe cierta controversia respecto a la realización de esfinterotomía endoscópica previa a la colocación de la endoprótesis biliar como factor de riesgo de la migración. Hay estudios que demuestran que no existe una relación significativa entre esfinterotomía endoscópica y migración ${ }^{13,16}$; mientras que Margulies ${ }^{17}$ señala la elevada frecuencia de migración distal en pacientes a los que no se les realizó esfinterotomía endoscópica, no influyendo ésta en la migración proximal.

La localización más frecuente de la endoprótesis biliar en la migración distal es el duodeno $0^{2,4-12}$. La mayoría de las endoprótesis que migran distalmente pasan por la luz intestinal sin complicaciones, expulsándose con las heces; aquellas que no pasan con normalidad por el intestino delgado o el colon, o cuya migración se detecta precozmente deben ser recuperadas por vía endoscópica y fluoroscópi$\mathrm{ca}^{2,18}$. En caso de no poder ser extraídas con estas técnicas, se aconseja realizar una cirugía precoz para evitar posibles complicaciones. Es raro que las endoprótesis biliares impacten en la pared intestinal, siendo la causa más frecuente de impactación la fijación extrínseca o irregularidades de la pared intestinal ${ }^{2,4,9,14}$, como el ligamento de Treitz ${ }^{19}$, las hernias paraestomales, las hernias abdominales, las adherencias intestinales y los divertículos colóni$\cos ^{20}$; una causa más rara sería el enclavamiento en el orificio apendicular ${ }^{21}$. Esto produciría perforaciones intestinales, sepsis intraabdominal, obstrucción intestinal, fístulas intestinales, perforación de divertículo colónico, fascitis necrotizante y apendicitis ${ }^{2}$. La localización más frecuente de perforación es el duodeno, debido al procedimiento endoscópico en sí o a la migración de la endoprótesis. Otros lugares de perforación son el colon, el íleon distal, el hígado y el páncreas ${ }^{8,14}$. Bagul $^{2}$ et al, tras revisar la literatura han observado que la mayoría de las complicaciones asociadas a la migración de las endoprótesis biliares y a la perforación se deben a endoprótesis plásticas rectas, como sucede en nuestro paciente; esto puede deberse al atrapamiento de la endoprótesis plástica en la pared intestinal, debido a las púas laterales que presenta la endoprótesis y a la fijación extrínseca o irregularidades de la pared intestinal, por lo que se produciría una necrosis por presión y posterior perforación intestinal.
Cuando un paciente tenga una endoprótesis biliar que haya emigrado por el tubo digestivo y que no pueda extraerse por vía endoscópica, debe indicarse una intervención quirúrgica precoz para evitar futuras complicaciones; en el caso de perforación intestinal la cirugía debe ser urgente, como en nuestro paciente, al que se le realizó extracción de la endoprótesis biliar y sutura primaria del divertículo colónico al no existir peritonitis, si existiera una contaminación intraabdominal se debería realizar la operación de Hartmann.

La perforación diverticular sigmoidea secundaria a la migración de una endoprótesis biliar plástica es una complicación rara, que puede aparecer tras la colocación de una endoprótesis plástica en la vía biliar y que debe sospecharse ante todo cuadro de abdomen agudo que se acompaña de imágenes radiológicas que demuestren la migración de la endoprótesis biliar, ya que estos pacientes no suelen presentar una sintomatología típica, lo que supondría un retraso diagnóstico y terapéutico perjudicial para el paciente.

\section{Referencias}

1. Chun HJ, Kim ES, Hyun JJ, Kwon YD, Keum B, Kim ChD. Gastrointestinal and biliary stents. J Gastroenterol Hepatol. 2010;25:234-43.

2. Bagul A, Pollard C, Dennison AR. A review of problems following insertion of biliary stents illustrated by an unusual complication. Ann R Coll Surg Engl. 2010 May 1; 92(4): W27-31. Disponible en: http://www.ingentaconnect.com/content/rcse/arcs/2010/00000092/00000004/ art00041 (Consultado el 10 de julio de 2012).

3. Soehendra N, Reynders-Frederix V. Palliative bile duct drainage: A new endoscopic method of introducing a transpapillary drain. Endoscopy 1980;12:8-11.

4. Namdar T, Raffel AM, Topp SA, Namdar L, Alldinger I, Schmitt M, et al. Complications and treatment of migrated biliary endoprostheses: A review of the literature. World Gastroenterol. 2007;13:5397-9.

5. Frakes JT, Johanson JF, Stake JJ. Optimal timing for stent replacement in malignant biliary tract obstruction. Gastrointest Endosc. 1993;39:164-7.

6. Pedersen FM, Lassen AT, Schaffalitzky de Muckadell OB. Randomized trial of stent placed above and across the sphincter of Oddi in malignant bile duct obstruction. Gastrointest Endosc. 1998;48:574-9.

7. Soto-Ávila JJ, Gómez-Wong ME, Preciado-Hernández F. Fístula colovesical secundaria a la migración de una endoprótesis biliar. Reporte de un caso. Cir Ciruj. 2006; 74:473-5.

8. Elliot M, Boland S. Sigmoid colon perforation following a migrated biliary stent. ANZ J Surg. 2003;73:669-70.

9. García-Figueiras R, Otero-Echart M, García-Figueiras 
A, Pazos-González G. Colocutaneous fistula relating to the migration of a biliary stent. Eur J Gastroenterol Hepatol. 2001;13:1251-3.

10. Lenzo NP, Garas G. Biliary stent migration with colonic diverticular perforation. Gastrointest Endosc. 1998;47:543-4.

11. Arhan M, Ödemiş B, Parlak E, Ertuğrul I, Başar Ö. Migration of biliary plastic stents: experience of a tertiary center. Surg Endosc. 2009;23:769-75.

12. Brinkley M, Wible BC, Hong K, Georgiades C. Colonic perforation by a percutaneously displaced biliary stent: Report of a case and a review of current practice. J Vasc Interv Radiol. 2009;20:680-3.

13. Johanson JF, Schmalz MJ, Geenen JE. Incidence and risk factors for biliary and pancreatic stent migration. Gastrointest Endosc. 1992;38:341-6.

14. Belyaev O, Müller CA, Uhl W. Double sigmoid colon perforation by a migrated biliary stent. Acta Chir Belg. 2008;108:125-6.

15. Diller R, Senninger N, Kautz G, Tübergen D. Stent migration necessitating surgical intervention. Surg Endosc. 2003;17:1803-7.

16. Giorgio PD, Luca LD. Comparison of treatment outco- mes between biliary plastic stent placements with and without endoscopic sphincterotomy for inoperable malignant common bile duct obstruction. World J Gastroenterol. 2004;10:1212-4.

17. Margulies C, Sampaio-Siqueira E, Silverman WB, Lin XS, Martin JA, Rabinovitz M, Slivka A. The effect of endoscopic sphincterotomy on acute and chronic complications of biliary endoprostheses. Gastrointest Endosc. 1999;49:716-9.

18. Cerisoli C, Diez J, Gimenez M, Oria M, Pardo R, Pujato M. Implantation of migrated biliary stents in the digestive tract. HPB 2003;5:180-2.

19. Culnan DM, Cicuto BJ, Singh H, Cherry RA. Percutaneous retrieval of a biliary stent after migration and ileal perforation. World J Emerg Surg. 2009;4:6.

20. Akbulut S, Cakabay B, Ozmen CA, Sezgin A, Sevinc MM. An unusual cause of ileal perforation: report of a case and literature review. World J Gastroenterol. 2009;15:2672-4.

21. Tzovaras G, Liakou P, Makryiannis E, Paroutoglou G. Acute appendicitis due to appendiceal obstruction from a migrated biliary stent. Am J Gastroenterol. 2007;102:195-6. 